\title{
Editorial: Issues Dedicated to Paul Erdős
}

A year ago we began a special year of issues dedicated to Paul Erdös on the occasion of his 80 th birthday. We were gratified to receive a large number of exceptional submissions, and to accommodate these we are extending our 'Erdös year' by two issues.

Cambridge University Press will shortly be publishing a special volume containing the papers submitted for the Cambridge Combinatorial Conference in Honour of Paul Erdős.

Béla Bollobás 